University of Wollongong

Research Online

Faculty of Engineering and Information

Faculty of Engineering and Information

Sciences - Papers: Part A

Sciences

$1-1-2014$

\title{
Influence of polarization and a source model for dose calculation in MRT
}

Stefan Bartzsch

The Institute of Cancer Research, Uk

Michael Lerch

University of Wollongong, mlerch@uow.edu.au

Marco Petasecca

University of Wollongong, marcop@uow.edu.au

Elke Brauer-Krisch

European Synchrotron Radiation Facility, brauer@esrf.fr

Uwe Oelfke

The Institute of Cancer Research, Uk

Follow this and additional works at: https://ro.uow.edu.au/eispapers

Part of the Engineering Commons, and the Science and Technology Studies Commons

Research Online is the open access institutional repository for the University of Wollongong. For further information contact the UOW Library: research-pubs@uow.edu.au 


\title{
Influence of polarization and a source model for dose calculation in MRT
}

\begin{abstract}
Purpose: Microbeam Radiation Therapy (MRT), an alternative preclinical treatment strategy using spatially modulated synchrotron radiation on a micrometer scale, has the great potential to cure malignant tumors (e.g., brain tumors) while having low side effects on normal tissue. Dose measurement and calculation in MRT is challenging because of the spatial accuracy required and the arising high dose differences. Dose calculation with Monte Carlo simulations is time consuming and their accuracy is still a matter of debate. In particular, the influence of photon polarization has been discussed in the literature. Moreover, it is controversial whether a complete knowledge of phase space trajectories, i.e., the simulation of the machine from the wiggler to the collimator, is necessary in order to accurately calculate the dose. Methods: With Monte Carlo simulations in the Geant4 toolkit, the authors investigate the influence of polarization on the dose distribution and the therapeutically important peak to valley dose ratios (PVDRs). Furthermore, the authors analyze in detail phase space information provided byMartínez-Rovira et al. ["Development and commissioning of a Monte Carlo photon model for the forthcoming clinical trials in microbeam radiation therapy," Med. Phys.39(1), 119-131 (2012)] and examine its influence on peak and valley doses. A simple source model is developed using parallel beams and its applicability is shown in a semiadjoint Monte Carlo simulation. Results are compared to measurements and previously published data. Results: Polarization has a significant influence on the scattered dose outside the microbeam field. In the radiation field, however, dose and PVDRs deduced from calculations without polarization and with polarization differ by less than $3 \%$. The authors show that the key consequences from the phase space information for dose calculations are inhomogeneous primary photon flux, partial absorption due to inclined beam incidence outside the field center, increased beam width and center to center distance due to the beam propagation from the collimator to the phantom surface and imperfect absorption in the absorber material of the Multislit Collimator. These corrections have an effect of approximately $10 \%$ on the valley dose and suffice to describe doses in MRT within the measurement uncertainties of currently available dosimetry techniques. Conclusions: The source for the first clinical pet trials in MRT is characterized with respect to its phase space and the photon polarization. The results suggest the use of a presented simplified phase space model in dose calculations and hence pave the way for alternative and fast dose calculation algorithms. They also show that the polarization is of minor importance for the clinical important peak and valley doses inside the microbeam field.
\end{abstract}

\section{Disciplines}

Engineering | Science and Technology Studies

\section{Publication Details}

Bartzsch, S., Lerch, M. LF., Petasecca, M., Brauer-Krisch, E. \& Oelfke, U. (2014). Influence of polarization and a source model for dose calculation in MRT. Medical Physics, 41 (4), 041703-1-041703-13. 


\title{
Influence of Polarisation and a source model for dose calculation in MRT
}

\author{
Stefan Bartzsch ${ }^{1,2}$, Michael Lerch ${ }^{3}$, Marco Petasecca ${ }^{3}$, Elke Bräuer-Krisch ${ }^{4}$, Uwe Oelfke ${ }^{1,2}$ \\ ${ }^{1}$ The Institute of Cancer Research, \\ 15 Cotswold Road, Belmont, Sutton, \\ Surrey SM2 5NG, United Kingdom \\ ${ }^{2}$ Deutsches Krebsforschungszentrum, \\ Im Neuenheimer Feld 280, D-69120 Heidelberg, Germany \\ ${ }^{3}$ Centre for Medical Radiation Physics, \\ University of Wollongong, Northfields Avenue, \\ Wollongong, 2522, Australia \\ and \\ ${ }^{4}$ European Synchrotron Radiation Facility, \\ 6 Rue Jules Horowitz, 38000 Grenoble, France
}

(Dated: February 13, 2014)

Introduction: Microbeam Radiation Therapy (MRT), an alternative preclinical treatment strategy using spatially modulated synchrotron radiation on a micrometre scale, has the great potential to cure malignant tumours (e.g. brain tumours) while having low side effects on normal tissue. Dose measurement and calculation in MRT is challenging because of the spatial accuracy required and the arising high dose differences. Dose calculation with Monte Carlo simulations are time consuming and their accuracy is still a matter of debate. In particular the influence of photon polarisation has been discussed in the literature. Moreover it is controversial whether a complete knowledge of phase space trajectories, i.e. the simulation of the machine from the wiggler to the collimator, is necessary in order to accurately calculate the dose.

Methods: With Monte Carlo simulations in the Geant4 toolkit we investigate the influence of polarisation on the dose distribution and the therapeutically important peak to valley dose ratios (PVDRs). Furthermore we analyse in detail phase space information provided by Martínez-Rovira et al. [1] and examine its influence on peak and valley doses. A simple source model is developed using parallel beams and its applicability is shown in a semi-adjoint Monte Carlo simulation. Results are compared to measurements and previously published data.

Results: Polarisation has a significant influence on the scattered dose outside the microbeam field. In the radiation field, however, dose and PVDRs deduced from calculations without polarisation and with polarisation differ by less than $3 \%$. We show that the key consequences from the phase space information for dose calculations are inhomogeneous primary photon flux, partial absorption due to inclined beam incidence outside the field centre, increased beam width and centre to centre distance (ctc) due to the beam propagation from the collimator to the phantom surface and imperfect absorption in the absorber material of the Multi Slit Collimator (MSC). These corrections have an effect of approximately $10 \%$ on the valley dose and suffice to describe doses in MRT within the measurement uncertainties of currently available dosimetry techniques. 


\section{INTRODUCTION}

Microbeam Radiation Therapy (MRT) is a novel preclinical tumour treatment strategy that uses arrays of narrow, a few micrometer wide beams separated by a few hundred micrometre spacing that have unusual high peak doses while the valley doses are assumed to stay beneath the tissue tolerance. Treatment is performed with high dose rates of up to $15,000 \mathrm{~Gy} / \mathrm{s}[2,3]$ to avoid dose blurring by respiration and vascular motion. So far only $3^{\text {rd }}$ generation synchrotrons such as the European Synchrotron Radiation Facility (ESRF) are capable of generating sufficiently high dose rates in the necessary energy domain of around $100 \mathrm{keV}$. Photon energies in this energy domain compromise sharp dose gradients at lower energies and high penetration depths at higher energies [4]. In preclinical studies it was shown that the tumour control of MRT is high while it spares normal tissue better than conventional therapies [5]. Laissue et al. [6] have shown that the mean survival time of rats baring frontocelebral 9L gliosarcoma was increased up to 7 times by microbeam treatment with 312 to 5000 Gy beam entrance doses, $100 \mu \mathrm{m}$ interbeam distance and $25 \mu \mathrm{m}$ beamwidth. In some cases the tumour even disappeared. The normal tissue, however, showed only minor damage to unidirectionally applied microbeams. The damage was confined to $25 \mu \mathrm{m}$ wide stripes exhibiting a loss of intersection areas of cross-firing experiments[6, 7].

After irradiation of healthy weanling piglets [8] with microbeams of beam entrance doses between 150 and 625 Gy, 20-30 $\mu \mathrm{m}$ beam width and $210 \mu \mathrm{m}$ spacing between the peaks no developmental abnormalities were observed compared to non-irradiated littermates in the up to 66 weeks follow up observation by qualified veterinary scientists. dangerous for the brain development. While sparing normal tissue MRT has the potential to even treat aggressive tumours such as squamous cell carcinomas. In preclinical studies Miura et al. [9] were able to show that the mean survival time of mice bearing this type of tumour could be significantly increased. The origin of the tissue sparing effect is commonly thought to be attributed to the replacement of endothelial cells lethally hit by endothelial cells the peak to valley dose ratio (PVDR) and a valley dose beneath the tissue tolerance dose.

A matter of debate are the optimal beam sizes and spacings. Serduc et al. [12] have found for 9L gliosarcomas in rat brains $50 \mu \mathrm{m}$ beam width and $400 \mu \mathrm{m}$ interbeam spacing as a good compromise between tumour control on the one hand side and normal tissue sparing on the other hand side. Irradiation fields were chosen to have always 18 Gy valley dose in the target. Dose calculation was performed with different Monte Carlo codes in the past, for example Geant4[13], EGS4[14, 15] and PENELOPE[16]. A code comparison for MRT was done by De Felici et al. [17].

For a long time microbeams have been assumed to be ideal parallel beams. Nettelbeck et al. [18] have as one of the first studied the influence of the collimator and the divergence of the beams. They found an increase in the penumbral dose of up to $26 \%$ when using divergent beams compared to ideal parallel microbeams. In the peak and midvalley, however, differences disappeared. Including inclined incidence on the collimator at the field edges produced up to $30 \%$ differences in the penumbra doses and lead to a variation of the full width half maximum (FWHM) of the microbeams of up to $4 \mu \mathrm{m}$. However, collimator scattering was found to be of no importance within the $2 \%$ uncertainties of the Monte Carlo simulations. Martínez-Rovira et al. [1] have performed a complete simulation of the beamline from the wiggler source down to the MSC. However, a detailed study of the influence of the gathered phase space information and the important parameters on the dose distribution in MRT is still missing.

Another particular property of synchrotron radiation is its linear polarisation. The polarisation effect has been a debate in the literature and its influence has not been totally clarified. Orion et al. [15] have for the first time used Compton and Rayleigh scattering cross sections (DCS) for polarised photons in an EGS4 Monte Carlo code for the dose calculation of microbeams. De Felici et al. [19] have used an EGS4 version to analyse the polarisation influence on PVDRs. They calculated $3 \times 3 \mathrm{~cm}^{2}$ microbeam fields with $25 \mu \mathrm{m}$ wide beams and $200 \mu \mathrm{m}$ spacing in a $16 \mathrm{~cm}$ long and $16 \mathrm{~cm}$ diameter water cylinder and found in 7 to $8 \mathrm{~cm}$ depth a difference between $(1 \pm 1) \%$ in the field centre, $(3 \pm 3) \%$ at the field edge and $(10 \pm 2) \%$ outside the field when rotating the polarisation direction by $90^{\circ}$. Hence they concluded that polarisation is of minor importance for therapy planning. They also investigated the asymmetric dose absorption around a pencil beam from 0 to $150 \mu \mathrm{m}$ distance from the beam centre and found between $7 \%$ and $18 \%$ direction depending differences. However, they did not analyse asymmetric dose absorption outside this range.

In the presented work we study the influence of the source model and the polarisation on the dose distribution in MRT in a water phantom. Results of Monte Carlo simulations are compared with dosimetric measurements. We develop a source model of parallel microbeams with corrections motivated by our analysis that shows agreement to dose calculations with complete source simulations. This model simplifies Monte Carlo dose calculations and facilitates 


\section{METHODS}

\section{A. Biomedical beamline ID17 at the ESRF}

The European Synchrotron radiation facility (ESRF) is a $3^{\text {rd }}$ generation synchrotron and one of the few radiation

100 sources in the world that are bright enough to enable MRT. It consists of a storage ring for $6.04 \mathrm{GeV}$ electrons and $200 \mathrm{~mA}$ current [20]. The present maximum photon beam size is $35 \times 100 \mathrm{~mm}^{2}$, but may be increased to $42 \times 100 \mathrm{~mm}$. For therapy a filtered white spectrum beam is used with a maximum intensity at $83 \mathrm{keV}$ and a mean energy of 100 $\mathrm{keV}$. The MSC is positioned $39.3 \mathrm{~m}$ from the x-ray wiggler source and ensures approximately parallel microbeams at the sample for a photon flux of still 16,000 Gy/s [21]. Between wiggler source and sample there are several beam modifier elements, such as filters $(\mathrm{C}, \mathrm{Al}$ and $\mathrm{Cu}$ ), a Be-window, an Al-window, primary slits, a horizontal and a vertical tungsten slit and finally the MSC that forms $50 \mu \mathrm{m}$ wide and $400 \mu \mathrm{m}$ spaced microbeams. This is the setup dedicated for the first clinical pet trials planned in November 2013. The collimator slits of the MSC are cut into an $8 \mathrm{~mm}$ thick tungsten carbide (WF20) block [21].

\section{B. Forward Monte Carlo calculations}

We have calculated the absorbed dose of microbeam fields in water using Monte Carlo simulations in the Geant4 tool-kit, version 4.9.3.p02. The Livermore low-energy polarized physics libraries were used because they take into account polarised photon interactions at low photon energies [22]. For photon interactions photoelectric absorption, Compton scattering, Gamma conversion and Rayleigh scattering are regarded. Activated interaction processes for electrons are multiple scattering, ionisation and bremsstrahlung. The dose was calculated with forward and adjoint Monte Carlo techniques using different source phase space assumptions that are explained in the following. In order to test the influence of the source model on the distribution of the absorbed dose we employed the phase space information, gathered and presented by Martínez-Rovira et al. [1]. The authors simulated the particle transport from the wiggler to a plane in front of the vertical slit at the ID17 beamline of the ESRF, $38.8 \mathrm{~m}$ downstream from the wiggler source (phase space plane) and used the Monte Carlo codes Shadow[23] and PENELOPE[24, 25] for their simulations. They created data files (phase space files, PSFs) for 3 different primary vertical slit apertures creating a primary beam of 1,2 or $3 \mathrm{~cm}$ width and $1 \mathrm{~mm}$ height in the phase space plane. For $1 \mathrm{~cm} \mathrm{beamwidth} 5.2 \cdot 10^{7}$, for $2 \mathrm{~cm} 6.3 \cdot 10^{7}$ and for $3 \mathrm{~cm} 12.5 \cdot 10^{7}$ phase space entries exist. Technical details on the simulations can be found in Martínez-Rovira et al. [1].

Comparisons are made between simulations with and without these phase space information and also with and without photon polarisation in order to investigate their influence on the dose distribution. Results are also compared to experimental data. Calculations employing the PSFs are performed in two steps. In the first step the phase space files described by Martínez-Rovira et al. [1] are used to create primary photons in the phase space plane. These photons are tracked through the vertical slit and the MSC. In a plane directly behind the MSC phase space information are stored again in a second PSF. In the second step the photons from the second PSF traverse $120 \mathrm{~cm}$ air until they impinge on a water phantom of 16x16x16 $\mathrm{cm}^{3}$ size. The dimensions correspond to the experimental set-up for dosimetry at the beamline. From the photons in the PSF of Martínez-Rovira et al. [1] the majority is absorbed in the MSC. The chosen approach has the advantage to efficiently reuse only those particles that passed the MSC. All forward Monte Carlo simulation results are obtained with $10^{10}$ simulated particle histories, a lot more than entries in the second PSF. This implies that statistical uncertainties present in the second PSF behind the MSC remain in the final results.

For simulations without phase space files the center-to-center distance (ctc) and width of the microbeams at the phantom surface are used. Microbeams are simulated as perfectly parallel beams with a rectangular profile, having $100 \%$ intensity in the beam and no intensity in the interbeam space (further denoted as ideal microbeams). From the divergence of the beam [1] one finds after $120 \mathrm{~cm}$ a ctc of $412.3 \mu \mathrm{m}$ and a width of $51.5 \mu \mathrm{m}$. Variations of this setup are used to analyse the reasons for deviations between ideal microbeams and microbeams simulated incorporating the phase space information.

\section{Semi-adjoint Monte Carlo simulation}

Adjoint Monte Carlo simulations are a useful Monte Carlo method if the detector size is small and the source size large in the phase space. In this case it is possible to exchange detector and source in the simulation and track particles backwards in time from the detector to the source. This modification is often associated with a reduction of statistical noise and can thus shorten calculation times. In some cases like for MRT it is desirable to exchange just 
certain dimensions of detector and source. To this method we refer as semi-adjoint Monte Carlo simulation. It is a modified method of the techniques that are for example described by Iván Lux [26]. Source and detector geometry are exchanged in a plane perpendicular to the beam propagation only, because in this plane the detector is small and the source large. The semi-adjoint Monte Carlo technique can be used to analyse dose distributions in lateral homogeneous phantoms. We have used it to analyse the effect of linear polarisation on the absorbed dose and to improve the statistics. We have simulated an infinitesimal small pencil beam in a water or solid water (RW3, [27]) phantom of $20 \times 30 \times 30 \mathrm{~cm}^{2}$ side length using $2.5 \cdot 10^{9}$ photons and scored the dose in a cylindrical symmetric detector around the beam as shown in figure 1 . This detector consists of ring segments, stacked in $\mathrm{z}$ covering $4^{\circ}$ in angle. The radial walls of the detector rings are situated at $r=\left(1.1^{i}\right) \mu m$ for $i=1,2, \ldots, 110$ yielding exponentially increasing detector volumes adapted to the steeply decreasing dose with distance from the beam axis.

The dose in water of a $3 \times 3 \mathrm{~cm}^{2}$ microbeam field with $400 \mu \mathrm{m}$ ctc and $50 \mu \mathrm{m}$ beam width was calculated in the semi-adjoint Monte Carlo method. The dose was determined on a Cartesian grid with $5 \mu$ m resolution perpendicular to the microbeams, $4 \mathrm{~mm}$ in the photon propagation direction and $1 \mathrm{~mm}$ parallel to the microbeams. To compare the polarisation effects on the dose outside the field with measurements we calculated in a similar way the dose in solid water (RW3) for a broad beam $2 \times 2 \mathrm{~cm}^{2}$ field in accordance to the experimental set-up at the ESRF, as described below.

\section{Dose measurements at the synchrotron}

The Monte Carlo simulation results obtained in the broadbeam configuration were benchmarked against equivalent biomedical beamline (ID17) at the European Synchrotron Radiation Facility in Grenoble, France. The synchrotron was operating with approximately $200 \mathrm{~mA}$ in the storage ring. All aspects (beam filters, wiggler gap size) of the experimental set-up were as for standard MRT conditions. A homogeneous X-ray field of $2 \times 2 \mathrm{~cm}^{2}$ was generated by moving the target at constant speed through the radiation beam of height $(0.051 \mathrm{~mm}$ or $0.52 \mathrm{~mm})$ and width $(20 \mathrm{~mm})$ as defined by the primary slit and a secondary slit. 170 The homogeneous beam was incident on the surface of a $30 \times 30 \mathrm{~cm}^{2}$ solid water phantom (as shown in the photo in figure 2(a)) approximately $42.9 \mathrm{~m}$ downstream of the wiggler insertion device. A PTW semiflex ionisation chamber (IC) was connected to a PTW UNIDOS (model T10002) electrometer for measurements and operated at 400 V. We followed the dosimetry protocol by the IAEA[28]. The semiflex IC was chosen due to its excellent response uniformity with incident photon energy within the photon energy range relevant for MRT, especially the out-of-field photon spectrum. Dose rate dependence is, however, a major challenge for the semiflex IC in MRT. However this dependence was minimised by creating the homogeneous X-ray field using a vertical slit height of $0.052 \mathrm{~mm}$ (mentioned above). If the slit height is smaller, the target can be moved at a reduced speed through the beam. This also decreases the effective dose rate seen by the IC, which is integrating over a larger volume. Previous measurements [29] have shown that the IC measurements are reliable with this slit size if an ion recombination correction factor (due to the high dose rate) of $6.3 \%$ is applied to in-field measurements. No such correction is required for out-of-field measurements as the dose rate is significantly less. The ionisation chamber was mounted in two orientations at several depths ( 5 to $120 \mathrm{~mm}$ ) of the solid water phantom as shown schematically in figure 2(b) to maintain the geometrical dimensions of the sensitive volume with respect to the field. In all measurements the thickness of backscatter material was constant $(8 \mathrm{~cm})$. The response the IC was measured at vertical and lateral distances of 0,2 , and $4 \mathrm{~cm}$ from the central axis of the radiation field. Other important parameters (e.g. ring current) were logged separately.

\section{RESULTS}

\section{A. Polarisation effects}

For a monoenergetic pencil beam of $150 \mathrm{keV}$ figure $3 \mathrm{~A}$ shows profiles perpendicular and parallel to the polarisation direction. Scattering of a photon perpendicular to its polarisation direction is more likely than parallel to it. In the profile there are clearly three domains in distance from the primary beam visible, separated by steep dose fall-offs (compare Spiga et al. [30]). The first domain is the region within $10 \mu \mathrm{m}$ around the beam. Photoelectric absorption and Compton scattering produce electrons in the central beam. At low energies the Compton electrons get just around $20 \%$ of the primary photon energy, whereas a photoelectron gets, neglecting binding energies, the whole photon energy. Hence the range of photoelectrons is much larger than that of Compton electrons. For a few tens of keV the electron range is in the order of a few microns, where as the electron CSDA range at $150 \mathrm{keV}$ is around 280 $\mu \mathrm{m}$ [31]. Energy absorption in the first $10 \mu \mathrm{m}$ around the central beam is therefore mediated by Compton electrons. Momentum conservation implies that electrons scatter off opposite to the scattered photons and hence they are mainly produced 
in the plane perpendicular to the polarisation. In a distance between 10 and $200 \mu \mathrm{m}$ from the beam the region of photoelectrons can be found. The photoelectric absorption is independent of the photon polarisation and hence there are no directional differences observed. Figure 3B shows the ratio between the profile perpendicular and parallel to the polarisation. In the second domain from 10 to $200 \mu \mathrm{m}$ the ratio is almost 1 whereas inside the $10 \mu \mathrm{m}$ radius a strong directional dependency can be seen. Electrons become maximal ionising at the end of their path. Hence at the end of the Compton electron range the polarisation effect is strongest and the ratio is peaked there.

Beyond $200 \mu \mathrm{m}$ energy absorption is mediated by scattered Compton photons. Primary photons are preferentially Bered perpendicular to their polarisation. The difference between the dose deposit perpendicular and parallel to the primary polarisation direction is around $60 \%$. This difference is almost independent of depth and distance from the beam. Only at very shallow depths the difference increases slightly with distance.

Looking at this data we expected a strong influence of the polarisation on the patient dose distribution in MRT. As described above the simulated pencil beam was used to calculate the dose in an MRT field of $3 \times 3 \mathrm{~cm}^{2}$ size, $400 \mu \mathrm{m}$ ctc and $50 \mu \mathrm{m}$ beam width. The result of the calculation is shown in Figure 4. The dose differences of calculations with and without polarisation are up to $45 \%$ outside the microbeam field (figure $4 \mathrm{~A}$ and $4 \mathrm{~B}$ ). Within the field, differences between polarised and non-polarised treatment appear in a narrow region at the microbeam penumbras (figure 4D). These regions have a width of around $20 \mu \mathrm{m}$ and differences can be as high as $9 \%$. They are caused by the polarisation sensitive Compton electrons and doses in the beam penumbras are lower for polarised photons. Differences in the peak doses are very small, though. The peak dose differences are smaller than $0.1 \%$ throughout the whole field. The valley dose difference between calculations with and without polarisation is lowest in the centre and increases towards the field edges. If polarisation is taken into account, valley dose values are a bit lower, but deviations remain below $3 \%$ for a $3 \times 3 \mathrm{~cm}^{2}$ field except for a $3 \mathrm{~mm}$ wide field edge. In the centre the polarisation has an effect of around $1 \%$. For other field sizes, e.g. a $2 \times 2 \mathrm{~cm}^{2}$ microbeam field deviations are very similar (results are not shown here).

In the beam setup at the ESRF in Grenoble the polarisation is perpendicular to the alignment of the microbeams. This means that scattering perpendicular to the microbeams in the direction of the valley regions is smaller than parallel to the microbeams. Amazingly, this does not necessarily lead to a constantly lower valley dose. In order to understand this effect we need to consider the microbeam field as a whole. The total scattering cross section does not depend on the photon radiation polarisation. Going from non-polarised to polarised photons only the distribution of isotropic but has a quadrupole structure. Its minima are placed on a line perpendicular to the microbeams and its maxima parallel to it. In a point in the centre of a large field the dose is determined by interactions homogeneously distributed around. Positive and negative parts of the quadrupoles cancel each other out and hence the absorbed dose does not differ from the dose of isotropically scattering non-polarised photons. Close to beam edges, there are photons the orientation of the edge is important. If the off-beam region is situated in the direction of the positive lobe of the quadrupole the decrease will be stronger, because the lacking energy of less back scattering particles is stronger. Whereas an off-beam region in direction of the negative lobe of the quadrupole implies a less pronounced dose decrease towards the beam edge, because particles from this direction did anyway contribute less to the dose.

In the peak dose region for instance there are only electron producing photon interactions along the microbeam. Perpendicular to the microbeam there are less interactions. The consequence is a slightly higher peak dose close to the peak edge and a dose reduction in the penumbra region just outside the beam. For the valley dose photon scattering is important. In the centre of the field there is an equilibrium of in and out-scattering photons. Close to the field edge there are less photons scattering in from a particular direction. Polarisation leads to an anisotropic lack of photons compared to non-polarised isotropic scattering. However, the average range of scattered photons is much larger than the field size. Therefore there is no dose increase towards the field edges visible but only the decrease outside the field moving perpendicular to the microbeam direction. Hence in the valley dose the effect leads to the relative differences shown in figure $4 \mathrm{C}(\mathrm{D})$.

\section{B. Comparison with measurements}

245

In the case of MRT the primary photon flux emerging from the superconducting wiggler is well known to be proportional to the current in the synchrotron storage ring. The dose rate was deduced by multiplying the measured dose by the ratio of the speed of the goniometer (typically $20-100 \mathrm{~mm} / \mathrm{sec}$ ) with the slit size that defines the height of the primary photon beam $(0.051 \mathrm{~mm}$ in this case). As such the dose rate is therefore more specifically the average dose rate for a point defined within the primary radiation field $\left(2 \times 2 \mathrm{~cm}^{2}\right.$ being the standard field size). The semiflex has a well documented significant dose rate dependence, so to minimise this dependence the height of the primary beam was reduced to $0.051 \mathrm{~mm}$ (usually $0.520 \mathrm{~mm}$ ) and independently checked within the primary field against a pin point IC (no such dose rate dependence) previously [29]. For all measurements made outside the primary radiation 
field, where the dose rate is orders of magnitude less than the within the primary field, the measured dose scaled within $1 \%$ in going between the 0.051 and $0.520 \mathrm{~mm}$ slits, which gives confidence to this data. Figure 5B shows the deduced dose rate per milliampere of storage ring current.

Figure 5A shows how the measured IC response falls off with distance up to $6 \mathrm{~cm}$ from the central axis in the vertical (solid lines) and horizontal (dashed lines) direction for four different depths $(0.5,2,4$ and $12 \mathrm{~cm})$. Each measurement at the positions and depths indicated were repeated three times and the measurement uncertainty for the $99 \%$ confidence limit is below $5 \%$ for all measurement points. The scattered energy deposited outside the $2 \times 2 \mathrm{~cm}^{2}$ from the central axis out to $6 \mathrm{~cm}$, appears to decrease with depth in the solid water phantom. This is due to the contribution to the energy deposition from forward scattered photons which increases with depth, in the case of the photon energy spectrum of the primary MRT radiation field. It can also be seen immediately that the presence of the polarised primary beam effects the vertical and horizontal scattered photon distribution (and hence dose delivered) all depths the vertical dose is consistently greater than the horizontal dose, which is consistent with that expected from theory and is in agreement with the MC simulations. Monte Carlo simulations conform to the measurements to less than $10 \%$ (except for 1 value). Some of the values lie outside the uncertainties provided by the measurement. However, the scattered dose in contrast to the peak doses depends heavily on collimator scattering and back scattering from surfaces surrounding the phantom that were not taken into account in the Monte Carlo calculations. Hence the agreement between Monte Carlo and calculation in the off-beam, low dose domain is reasonably good.

\section{Source Phase Space}

PVDRs calculated with Monte Carlo simulations regarding the phase space information are on average $8 \%$ smaller than simulations assuming ideal microbeams. This result can be seen in Figure 8C. Although peak doses in the field centre are equal in both simulations, valley doses are substantially lower in calculations with ideal microbeams. It remains however unclear, where these differences originate from. In the following we investigate the physical and technical reasons for these deviations. The analysis was done in the PSF in the phase space plane and in the second PSF created directly behind the collimator. In the following $\mathrm{y}$ is the propagation direction of the photons, $\mathrm{z}$ the direction parallel to the collimator slits and $\mathrm{x}$ perpendicular to it. The direction of the photon momentum is denoted as $\vec{u}=\left(u_{x}, u_{y}, u_{z}\right)$ with $|\vec{u}|=1$. We concentrated on the following deviations from ideal microbeams and show that they explain the deviations observed:

1. Geometrical beam divergence of the whole field: Although the collimator is in a distance of $39.3 \mathrm{~m}$ from the wiggler source and the field is just a few centimetre in size, there is a measurable geometrical divergence. One consequence is that at the phantom surface $1.2 \mathrm{~m}$ from the collimator ctc and width of the microbeams have slightly increased.

2. Imperfect absorption of the absorber material in the tungsten carbide collimator leads to a leakage radiation.

3. Associated with the geometrical divergence could be a more pronounced absorption at the MSC for beams at the field edge, because the beam incidence is inclined towards the collimator walls.

4. The photon fluence is not completely flat. I.e. the photon flux prior to the MSC is higher at the centre than at the beam edges.

To estimate the error made by the neglect of the geometrical beam divergence we have plotted phase space points over $x$ and $u_{x}$ in figure $6 \mathrm{~A}$. All phase space points are more or less aligned along a straight line. As shown before by Martínez-Rovira et al. [1] the linear dependence between $x$ and $u_{x}$ with the regression coefficient $\gamma$ is given by

$$
u_{x}=\gamma x=2.56(4) \cdot 10^{-5} \mathrm{~mm}^{-1} x .
$$

The beam divergence remains unchanged after the photons have passed the MSC. The consequence of this divergence is that the beam width of the microbeams has increased from $50 \mu \mathrm{m}$ to $51.5 \mu \mathrm{m}$ and the ctc from $400 \mu \mathrm{m}$ to 412.3 $\mu \mathrm{m}$ when reaching the phantom surface in $120 \mathrm{~cm}$ distance from the MSC. The divergence itself inside the phantom is of negligible importance. At the back side of a $20 \mathrm{~cm}$ thick phantom the beam parameters have not significantly changed and are 51.8 and $414.4 \mu \mathrm{m}$. This difference is hardly measurable and is unlikely to have any clinical impact.

Apart from this over all geometric divergence there is an intrinsic beam divergence emanating from collimator scattering superimposing the geometrical divergence. Figure $6 \mathrm{~B}$ shows the dependence of $x$ and $u_{x}$ in the centre of the field in more detail. The actual trajectories in the phase space scatter around the found linear relation. Each 
beam has an intrinsic divergence which is given by $u_{x}=2 \cdot 10^{-6}$ in the centre of the field and $4.8 \cdot 10^{-6}$ at the field edges. The consequence for the beam shape at the phantoms surface in $120 \mathrm{~cm}$ distance from the collimator is that beam sizes and spacing are much higher than that, there is no substantial influence on the absolute peak and valley dose values to be expected. Therefore the valley dose contribution from collimator scattering is small enough to be neglected.

Higher valley doses, however, could also be a result of imperfect absorption from the collimator material. The photon flux behind the absorber material is around $1.0 \cdot 10^{-3}$ of the flux behind the aperture. However, the average energy of the photons behind the absorber material is about 2.4 times higher than the average photon energy behind the apertures. Figure $6 \mathrm{C}$ shows the average photon energy depending on the position $(\mathrm{x})$ in a region close to the centre of the field. The average energy in the beam is around $100 \mathrm{keV}$. In the off-beam region the average photon energy is $240 \mathrm{keV}$. Due to less particles penetrating the absorber the noise level is higher than in the peaks. The collimator material is a tungsten carbide alloy (WF20) containing $39.41 \%$ tungsten, $40.72 \%$ carbon, $18.11 \%$ Co, $0.44 \%$ vanadium and $1.32 \%$ chrome [21]. Its thickness is $8 \mathrm{~mm}$. In order to confirm that the photon flux behind the collimator is caused by photons of the primary beam passing through the absorber we have calculated the spectral change of the beam after penetrating $8 \mathrm{~mm}$ WF20 and compared it with the actual spectrum behind the absorber material in Figure 6D. The red curve shows the MRT beam spectrum and the black dashed line is the derived spectrum after the beam has penetrated $8 \mathrm{~mm}$ of WF20. The actual observed spectrum is shown with blue plus symbols. The maxima of the spectra are normalised to 1. Calculated and observed spectrum behind the absorber coincide except for energies beneath around $135 \mathrm{keV}$. Their energy contribution of photons with less than $135 \mathrm{keV}$ is $1.35 \%$ and can probably be attributed to scattering at the collimator edges. Its small contribution consolidates the finding that collimator scattering into the valley region is of minor importance. Furthermore the divergence of the beam behind the absorber matches that of the microbeams as can be seen from figure $6 \mathrm{~B}$. So even after penetrating $8 \mathrm{~mm}$ absorber material the divergence of the beam has not increased.

In the plane directly behind the MSC the flux per microbeam changes depending on the position of the beam. The flux is highest in the centre of the field and decreases by about $9 \%$ to the edge of the field (see figure $7 \mathrm{~B}$, blue ' + '). This deviation consists of two parts. On the one hand side the photon flux in the phase space plane is smaller at the field edges than in the field centre. This is shown in figure 7A. The photon flux follows the quadratic profile

$$
I=I_{0}\left(1-0.0196 \mathrm{~cm}^{-2} x^{2}\right),
$$

where $I_{0}$ is the maximum dose in the centre of the field. The caused variations in the photon flux per beam are around $4 \%$. On the other hand side the inclined incidence of the beam outside the field centre leads to a partial shielding of the microbeams. Assuming the MSC made of ideal absorbing material (absorption coefficient $\mu \rightarrow \infty$ ) simple geometrical considerations yield for the ratio of actually transmitted photons $N$ to the number of transmitted photons in the field centre $N_{0}$

$$
\frac{N}{N_{0}}=1-\frac{\gamma(\text { absorber_thickness) }}{\text { (beam_width) }}|x|=1-\beta|x| .
$$

Using the linear divergence $\gamma=2.564 \cdot 10^{-5} \mathrm{~mm}$, the absorber thickness of $8 \mathrm{~mm}$ and the beam width of $50 \mu \mathrm{m}$ gives $\beta$ $=0.041 \mathrm{~cm}^{-2}$. However, due to imperfect absorption at the collimator edges the actually observed value is $\beta=0.030$ $\mathrm{cm}^{-1}$. From the centre to the edge the number of photons per beam decreases by $5 \%$ due to this effect. Taking both effects together the photon numbers per beam can accurately be described as shown in figure $7 \mathrm{~B}$. The total variations of peak doses are around $9 \%$ of its maximum.

Summarizing these findings we established the following simplified source model:

1. Parallel microbeams of $51.5 \mu \mathrm{m}$ width and $412.3 \mu \mathrm{m}$ spacing comprised of photons with energies according to the ESRF spectrum impinge the sample.

2. Parallel leakage radiation with an altered spectrum in the spacing between the beams contributes to the valley dose. The photon flux is reduced to $0.10 \%$ of the primary beam.

3. The photon flux in the beam varies with the beam position according to $I_{0}\left(1-\alpha x^{2}-\beta|x|\right)$ with the constants $\alpha=0.0196 \mathrm{~cm}^{-2}$ and $\beta=0.030 \mathrm{~cm}^{-1}$.

4. The leakage radiation follows the profile of the primary beam in the phase space plane, $I_{0}\left(1-\alpha x^{2}\right)$.

This simple source model can easily be implemented in Monte Carlo simulations, but also in other dose calculation techniques. In kernel based dose calculation algorithms the primary photon fluence of the field can be adopted with changed width and ctc of the microbeam and modulated photon beam intensity as described in point (3). The 
correction for the leakage dose can easily be performed by calculating the photon absorption of a broad parallel field with $0.1 \%$ flux of the primary field with the spectrum shown in figure $6 \mathrm{D}$. In the following we want to describe the usage of the found source model in semi-adjoint Monte Carlo simulations and compare its results with a forward calculation using the complete phase space information.

\section{Semi-Adjoint Monte Carlo simulation}

The developed source model can be employed in a semi-adjoint Monte Carlo simulation based on the principles of the phase space by (there are no directional constraints)

$$
\begin{aligned}
D(x, y, z, E)= & \Theta_{X Z}(x, z) \Delta\left(y-y_{0}\right) \\
& \left(\Theta(w-x \bmod a)\left(1-\alpha x^{2}-\beta|x|\right)+\Theta(x \bmod a-w)\left(1-\alpha x^{2}\right) \rho_{A b s}(E)\right) .
\end{aligned}
$$

Here we use $\mathrm{E}$ as the photon energy, $\mathrm{x}, \mathrm{y}$ and $\mathrm{z}$ are the coordinates as defined before, $w=51.5 \mu \mathrm{m}$ is the beam width, $a=412.3 \mu \mathrm{m}$ the centre-to-centre distance of the beams, $\mathrm{d}$ the depth of the detector in water and $\rho_{A b s}$ characterises the transmission of the primary photons through the absorber material. It is defined as number of photons $\mathrm{N}$ with energy $\mathrm{E}$ that penetrate the absorber per number of primary photons $N_{0}$ with energy $\mathrm{E}, \rho_{A b s}(E)=e^{-\mu_{W F 20}(E) \cdot d}$, with $\mathrm{d}=8 \mathrm{~mm}$ being the absorber thickness and $\mu_{W F 20}$ the absorption coefficient of tungsten carbide. The operator mod stands for modulo (remainder of division), the function $\Theta$ is one for positive arguments and zero otherwise; and the function $\Theta_{X Z}$ is 1 in the microbeam field and 0 otherwise. $\Delta\left(y-y_{0}\right)$ is one in a region around $y=y_{0}$ and characterises the sampling in y. For the simulations presented here the mesh size in y was chosen to be $4 \mathrm{~mm}$, i.e. $\Delta\left(y-y_{0}\right)$ is one for $y \in\left[y_{0}-2 \mathrm{~mm}, y_{0}+2 \mathrm{~mm}\right]$ and zero otherwise. The adjoint source is a point source at the surface of the phantom sending a parallel photon pencil beam into the phantom. The photon energy is distributed according to the spectrum of the primary beam (red line in figure 6D).

The results of this calculation can be seen in figure 8 . Figure 8 compares profiles in $8 \mathrm{~cm}$ depth of forward Monte Carlo simulations with PSF and adjoint Monte Carlo using the described detector. In figures $8 \mathrm{~A}$ and $8 \mathrm{~B}$ profiles of the microbeam field are shown in the field centre. Although the forward calculation used 10 times more particles, the statistical uncertainties are much higher than in the adjoint MC. Within uncertainties both methods, adjoint Monte Carlo simulation and forward Monte Carlo simulation, provide the same results.

In figure $8 \mathrm{C}$ the PVDR is compared between Monte Carlo simulations with the PSF (red ' ''), adjoint Monte Carlo simulation (blue line) and a Monte Carlo simulation with ideal microbeams (green squares). Figure 8D shows the valley doses for the same three calculations. The adjoint Monte Carlo coincides with the forward calculation with the PSF. The valley dose for the forward calculation with ideal microbeams is significantly lower. This difference originates from the neglect of leakage photons in the valley region. The peak dose also shows a modulation across the beam (see figure 8A). Moving out from the field centre peak doses calculated with our phase space model or the PSFs from Martínez-Rovira et al. [1] decrease where as the peak dose for ideal microbeams remains approximately constant.

\section{E. Comparisons with measurements and unexplained deviations}

In figure 9 results of the adjoint Monte Carlo simulation are compared with film dosimetry measurements and Monte Carlo simulations from Martínez-Rovira et al. [1]. The comparison is made for a $60 \times 24 \times 60 \mathrm{~cm}^{3}$ solid water phantom for a 20x20 and a 30x30 $\mathrm{cm}^{2}$ microbeam field. The results obtained with our model lie for most of the measurement points within the uncertainty limits of the experimental data. At shallow depth for th $30 x 30 \mathrm{~cm}^{2}$ field PVDRs tend to be slightly lower in the measurement. However, uncertainties of the film dosimetry are too high and more accurate measurements need to be performed to validate this effect. Compared to the Monte Carlo simulations by Martínez-Rovira et al. [1] our calculations seem to be closer to the measurement. The origin of these differences has not yet been resolved.

\section{CONCLUSIONS}

The characterisation of the source accomplished by Martínez-Rovira et al. [1] is the basis for the development of a treatment planning system. With the aid of their developed phase space information it was possible to find the key features influencing the dose distribution in MRT. They showed that the approximation of ideal microbeams is not 
broad beam, the insufficient absorption of the absorber material and the geometrical change of the beam width due to inclined incidence outside the beam axis. Regarding these parameters it is possible to work with parallel beams if the beam width and the ctc are corrected according to the divergence of the microbeam field and the distance between collimator and phantom surface. Divergence due to collimator scattering, however, seems to be of minor importance. simulations. We have shown exemplarily the usage of the semi-adjoint Monte Carlo technique and compared the results with forward calculations. Peak and valley doses are equal for Monte Carlo simulations using the complete phase space information and a semi-adjoint Monte Carlo method implementing the model we propose.

Of course there are several short comings in the developed model. Intrinsic beam divergence and collimator scatterour model does not account for variations in the spectrum between individual microbeams, e.g. because of partial absorption for inclined incidence at the field edges and is ignoring scattering in the air between MSC and phantom. All calculations are performed assuming perfectly plane surfaces in the collimators. Possibly photon scattering on rough surfaces or impurities could contribute to the dose distribution at the sample or patient position. The narrow However, looking at the available dosimetry techniques it is not yet possible to conclude on the influence of these effects, but promising research is in progress to make this possible in the near future [2,32].

In general the polarisation of synchrotron radiation and the consequently anisotropic Compton scattering needs to be regarded in order to achieve appropriate dose estimates. Especially in the off-field region polarisation has an domain of highest therapeutic interest they are less than $3 \%$. Only the shape of the beam penumbra changes slightly. This finding justifies the calculation of PVDRs and valley doses in the field neglecting the photon polarisation.

This study provides a crucial analysis comparing computational approaches with and without PSFs and polarisation. The presented data will on the one side increase the accuracy of the therapy planning system that will be used for our findings are a valuable information for the MRT research community, that often used simplified Monte Carlo calculations in preclinical experiments in the past. Calculations performed without the entire phase space information will have a difference in the valley dose of approximately $10 \%$. Ignoring the polarisation of the beam is only of minor importance in the centre of the field and corrections here are in the order of 1-3\%. Thus, the interpretation of the of biological understanding in MRT this is probably not a matter of concern for the first clinical pet trials.

\section{REFERENCES}

[1] I. Martínez-Rovira, J. Sempau, and Y. Prezado, Med. Phys. 39 (1) (2012).

[2] M. Petasecca, A. Cullen, I. Fuduli, A. Espinoza, C. Porumb, C. Stanton, A. H. Aldosari, E. Bräuer-Krisch, H. Requardt, A. Bravin, V. Perevertaylo, A. B. Rosenfeld, and M. L. F. Lerch, Journal of Instrumentation 7, P07022 (2012).

[3] E. Bräuer-Krisch, A. Bravin, M. Lerch, A. Rosenfeld, J. Stepanek, M. D. Michiel, and J. A. Laissue, Medical Physics 30, $583(2003)$.

[4] D. N. Slatkin, P. Spanne, F. A. Dilmanian, and M. Sandborg, Med. Phys. 19 (6) (1992).

[5] E. Bräuer-Krisch, R. Serduc, E. A. Siegbahn, G. L. Duc, Y. Prezado, A. Bravin, H. Blattmann, and J. A. Laissue, Mutation Research (2010).

[6] J. A. Laissue, G. Geiser, P. O. Spanne, F. A. Dilmanian, J.-O. Gebbers, N. Geiser, X. Y. Wu, M. S. Makar, P. L. Micca, M. M. Nawrocky, D. D. Joel, and D. N. Slatkin, Int. J. Cancer 78, 654 (1998).

[7] J. A. Laissue, N. Lyubimova, H. P. Wagner, D. W. Archer, D. N. S. andM. Di Michiel, C. Nemoz, M. Renier, E. Brauer, P. O. Spanne, J. O.Gebbers, K. Dixon, and H. Blattmann, Proc. SPIE 3770, 38-45 (1999).

[8] J. A. Laissue, H. Blattmann, M. D. Michiel, D. N. Slatkin, N. Lyubimova, R. Guzman, W. Zimmermann, S. Birrer, T. Bley, P. Kircher, R. Stettler, R. Fatzer, A. Jaggy, H. Smilowitz, E. Bräuer-Krisch, A. Bravin, G. L. Duc, C. Nemoz, M. Renier, W. C. Thomlinson, J. Stepanek, and H.-P. Wagner, Proc. SPIE 4508, 65-73 (2001).

[9] M. Miura, H. Blattmann, E. Brauer-Krisch, A. Bravin, A. L. Hanson, M. M. Nawrocky, P. L. Micca, D. N. Slatkin, and J. A. Laissue, Br. J. Radiol. 79, 71-75 (2006). 
[11] H. Blattmann, J.-O. Gebbers, E. Bräuer, A. Bravin, G. L. Duc, W. Burkard, M. D. Michiel, V. Djonov, D. N. Slatkin, J. Stepanek, and J. A. Laissue, Nucl. Instrum. Methods Phys. Res. A 548, 17-22 (2005).

[12] R. Serduc, A. Bouchet, E. Bräuer-Krisch, J. A. Laissue, S. S. Jenny Spiga, C. F. Alberto Bravin, L. Renaud, J. Boutonnat, E. A. Siegbahn, F. Estève, and G. L. Duc, Physics in Medicine and Biology 54, 6711 (2009).

[13] J. Stepanek, H. Blattmann, J. A. Laissue, N. Lyubimova, M. D. Michiel, and D. N. Slatkin, Medical Physics 27, 1664 (2000).

[14] M. De Felici, R. Felici, C. Ferrero, A. Bravin, A. Tartari, and M. Gambaccini, Nuclear Instruments and Methods in Physics Research Section A: Accelerators, Spectrometers, Detectors and Associated Equipment 580, 489-492 (2007).

[15] I. Orion, A. B. Rosenfeld, F. A. Dilmanian, F. Telang, B. Ren, and Y. Namito, Phys. Med. Biol. 45, 2497-2508 (2000).

[16] E. A. Siegbahn, J. Stepanek, E. Bräuer-Krisch, and A. Bravin, Medical Physics 33, 3248 (2006).

[17] M. De Felici, E. A. Siegbahn, J. Spiga, A. L. Hanson, R. Felici, C. Ferrero, A. Tartari, M. Gambaccini, J. Keyriläinen, and E. Bräuer-Krisch, in Journal of Physics: Conference Series, Vol. 102 (2008) p. 012005.

[18] H. Nettelbeck, G. J. Takacs, M. L. F. Lerch, and A. B. Rosenfeld, Med. Phys. 36 (2) (2009).

[20] "European synchrotron radiation facility," (2013), http://www.esrf.eu/.

[21] E. Bräuer-Krisch, H. Requardt, T. Brochard, G. Berruyer, M. Renier, J. A. Laissue, and A. Bravin, Rev. Sci. Instrum. 80, 074301 (2009).

[22] Geant4-Collaboration, (2014), geant4 Internet Documentation, http://www.geant4.org.

[23] B. Lai and F. Cerrina, Nuclear Instruments and Methods in Physics Research Section A: Accelerators, Spectrometers, Detectors and Associated Equipment 246, 337-341 (1986).

[24] J. Baro, J. Sempau, J. M. Fernández-Varea, and F. Salvat, Nuclear Instruments and Methods in Physics Research Section B: Beam Interactions with Materials and Atoms 100, 31-46 (1995).

[25] F. Salvat, J. M. Fernández-Varea, and J. Sempau, in Workshop Proceedings, Vol. 4 (2006) p. 7.

[26] L. K. Iván Lux, Monte Carlo PARTICLE Transport Methods: Neutron and Photon Calculations (CRC Press, 2000).

[27] R. Hill, S. Brown, and C. Baldock, Radiation Measurements 43, 1258 (2008).

[28] International Atomic Energy Agency, "TRS-398," (2001).

[29] E. Bräuer-Krisch, University College London PhD thesis (2012).

[30] J. Spiga, E. A. Siegbahn, E. B. Krisch, P. Randaccio, and A. Bravin, Med. Phys. 34, 4322 (2007).

[31] M. Berger, J. Hubbel, S. Steltzer, J. Chang, J. Coursey, R. Sukumar, and D. Zucker, "Xcom: photon cross section database," National Institute of Standards and Technology, Gaithersburg, MD (2010), (version 3.1) [online available: http://www.nist.gov/pml/data/xcom/index.cfm, March 2011].

[32] M. Lerch, M. Petasecca, A. Cullen, A. Hamad, H. Requardt, E. Bräuer-Krisch, A. Bravin, V. Perevertaylo, and A. Rosenfeld, Radiation Measurements 46, 1560 (2011).

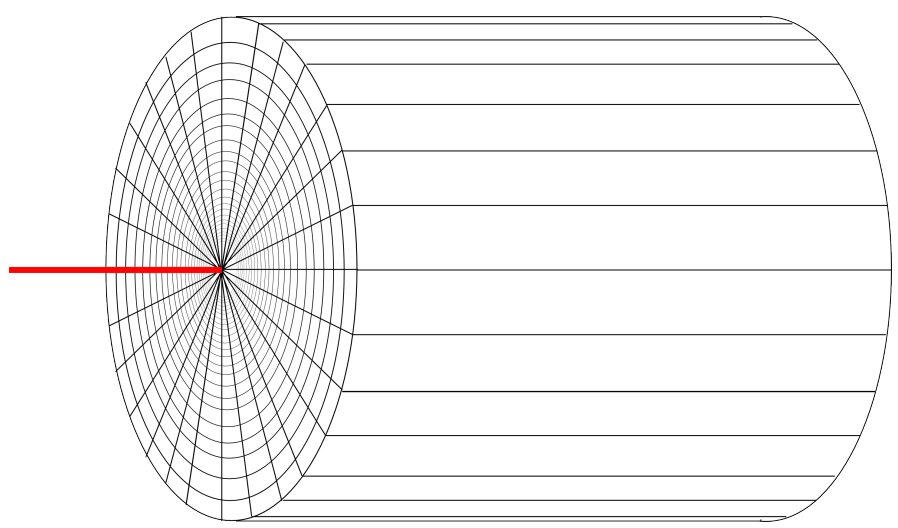

Figure 1 - Detector geometry to score an infinitely small microbeam in homogeneous water 
A

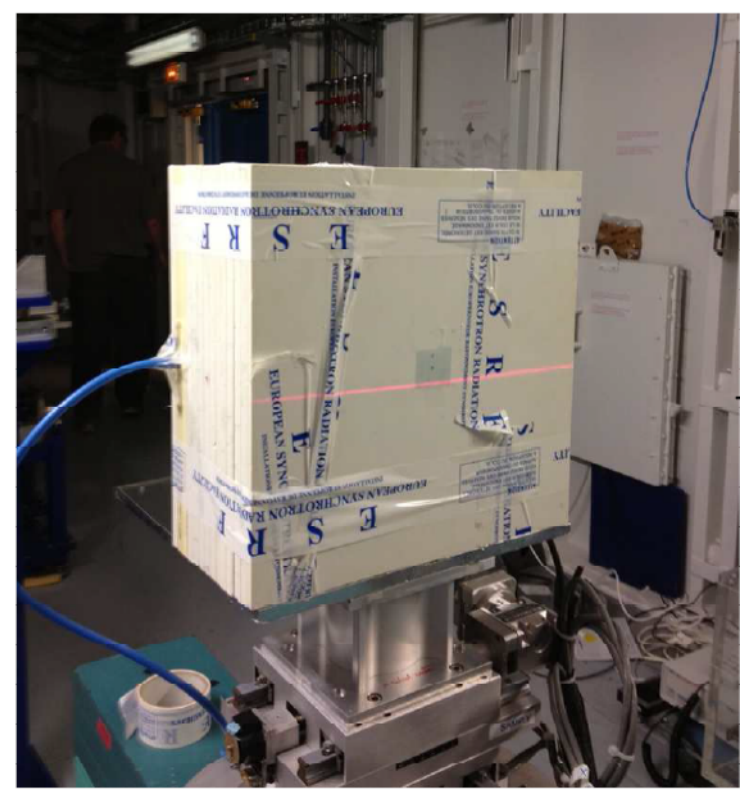

B

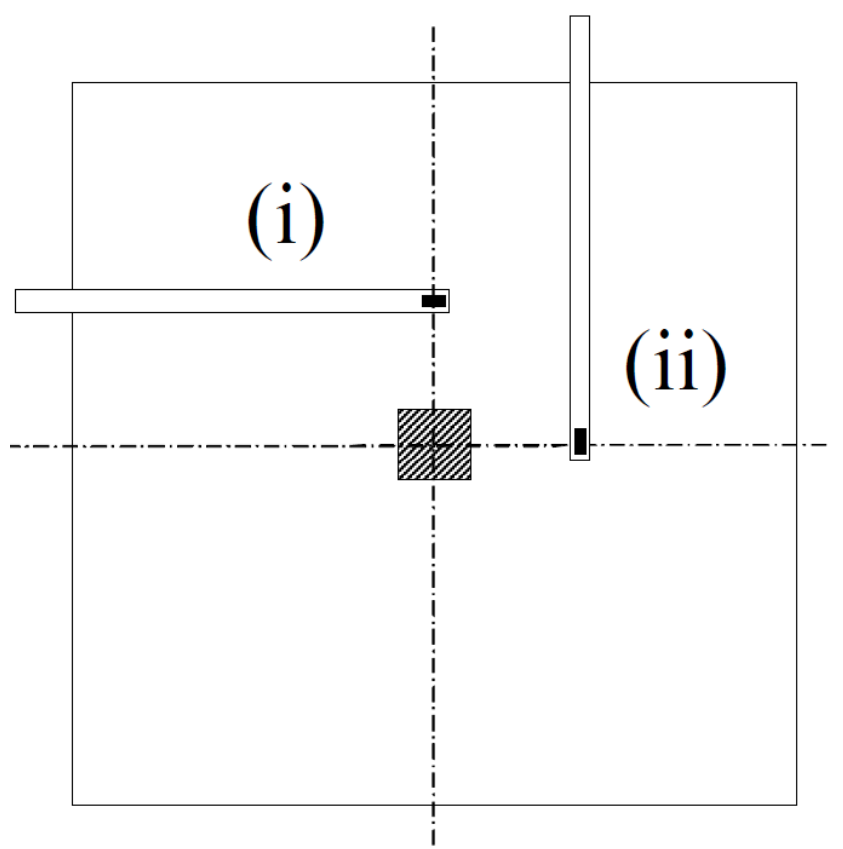

Figure 2 - A: Photo of the experiment set-up at the ESRF. B: Schematic diagram showing the orientation of the ionisation chamber for measuring the (i) vertical and (ii) lateral out-of-field dose components. Radiation field is indicated by the hashed region.

A

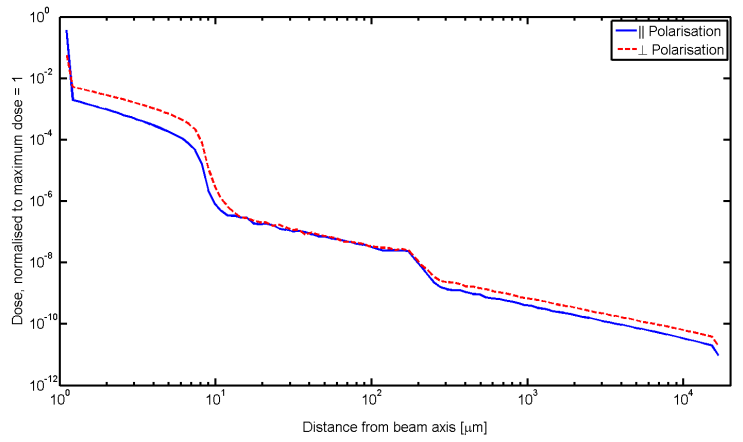

$\mathrm{B}$

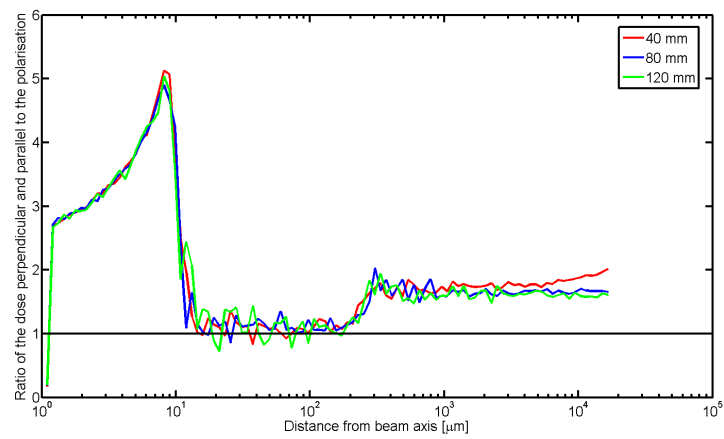

Figure 3 - A shows the lateral dose profile from the beam axis parallel and perpendicular to the polarisation direction for a photon pencilbeam of $150 \mathrm{keV}$. In $\mathrm{B}$ the ratio between the profile perpendicular to the polarisation direction and parallel to the polarisation direction is plotted. There are 3 different regions distinguishable. 
A

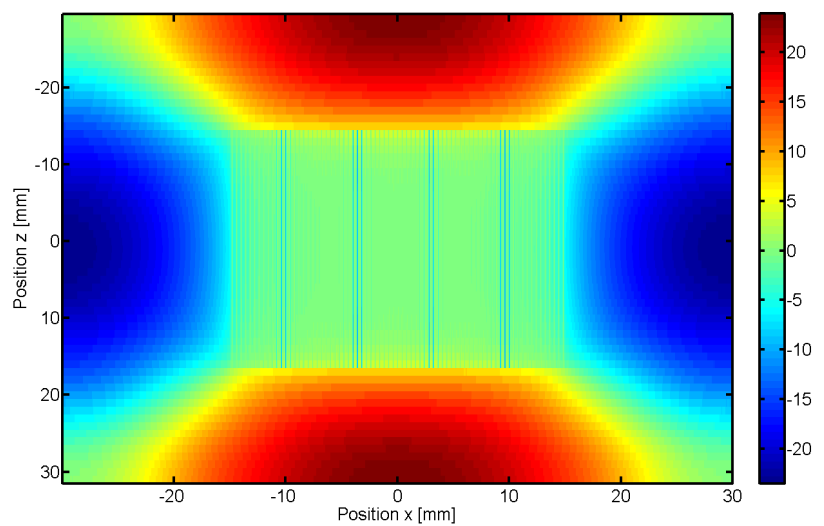

$\mathrm{C}$

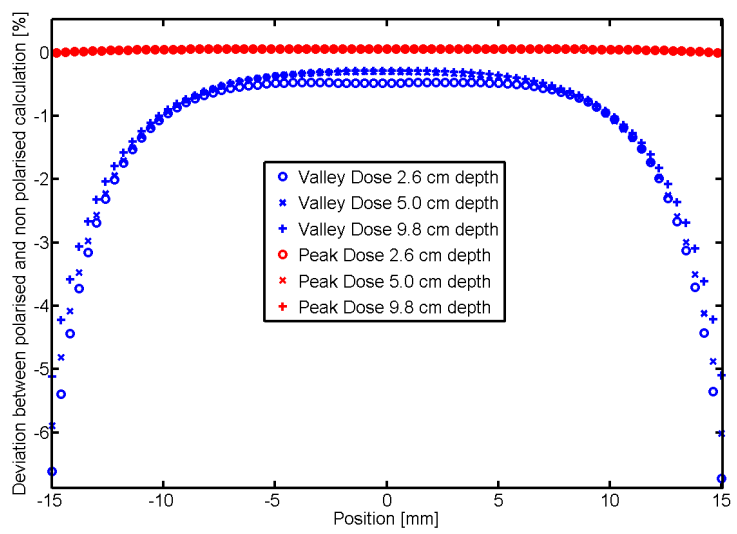

$\mathrm{B}$

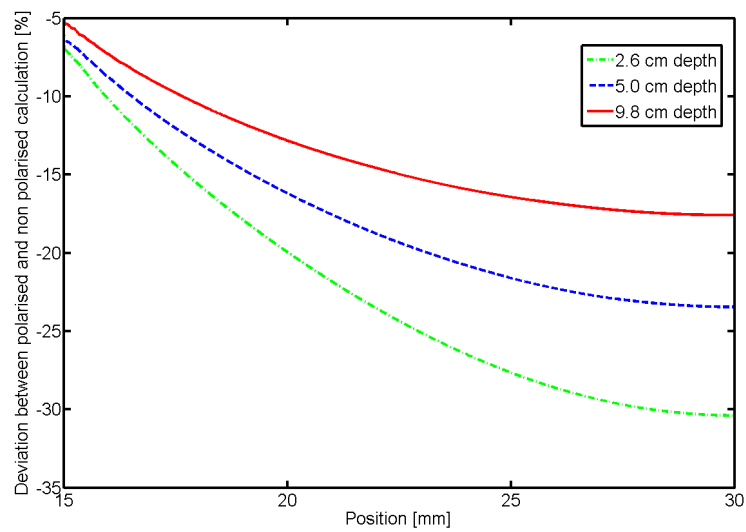

$\mathrm{D}$

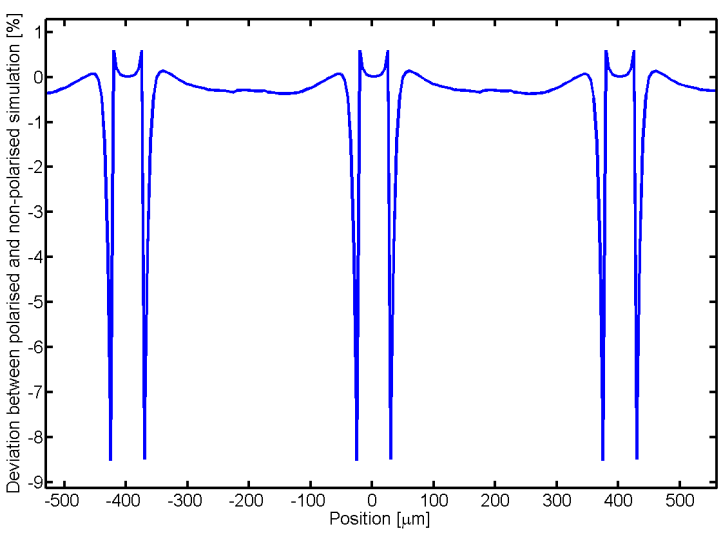

Figure 4 - Differences between dose calculations regarding and ignoring photon polarisation in a $3 \times 3 \mathrm{~cm}^{2}$ microbeam field in a water box. It is positive if the dose of a calculation with polarised photons is higher. Figure A shows the whole field in $5 \mathrm{~cm}$ depth. The colour coding states the differences in \%. Figure B shows differences at different depths outside the microbeam field and figure C compares peak and valley doses at the same dephts. In figure D differences in the centre of the field for 3 adjacent microbeams are shown. Except for the overshoot in the beam penumbra difference are very small. 
A

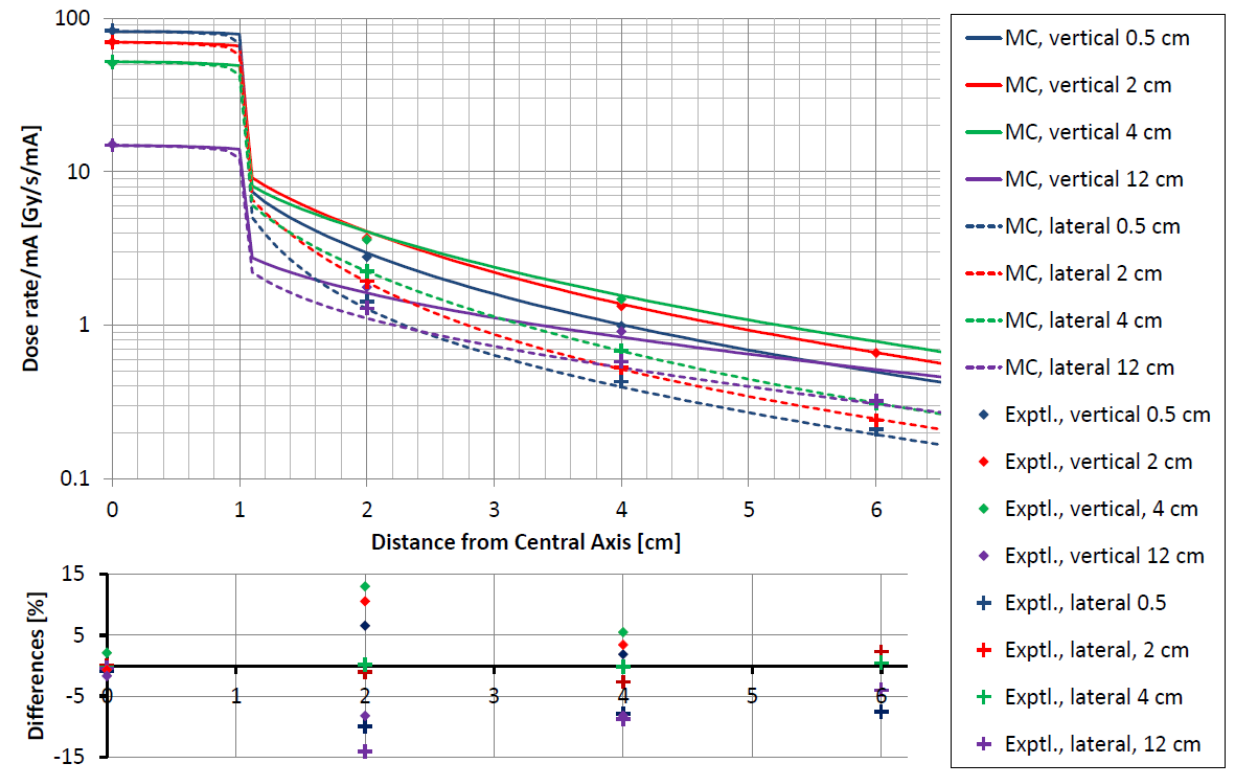

B

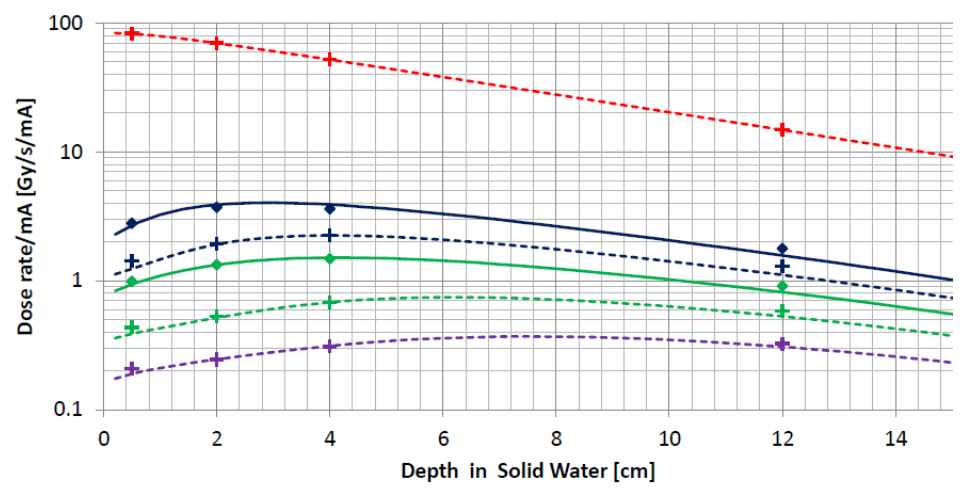

- Exptl., vertical at $2 \mathrm{~cm}$

- Exptl., vertical at $4 \mathrm{~cm}$

+ Exptl., lateral at $0 \mathrm{~cm}$

+ Exptl., lateral at $2 \mathrm{~cm}$

+ Exptl., lateral at $4 \mathrm{~cm}$

+ Exptl., lateral at $6 \mathrm{~cm}$

$-\mathrm{MC}$, vertical at $4 \mathrm{~cm}$

- MC, vertical at $2 \mathrm{~cm}$

$\ldots \mathrm{MC}$, lateral at $0 \mathrm{~cm}$

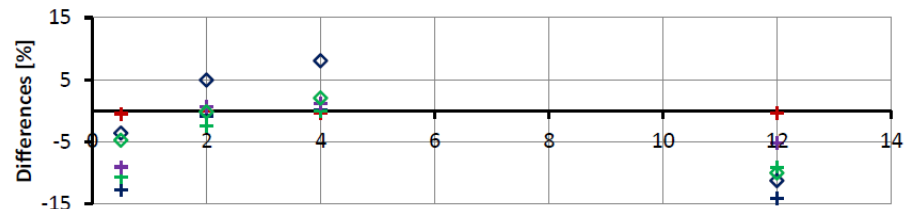

-- MC, lateral at $2 \mathrm{~cm}$

--- MC, lateral at $4 \mathrm{~cm}$

--- MC, lateral at $6 \mathrm{~cm}$

Figure 5 - A: Dose rate per $\mathrm{mA}$ of storage current deduced from measured IC response falls off with distance up to $6 \mathrm{~cm}$ from the central axis in the vertical (diamonds) and lateral (crosses) direction for the three different depths indicated. Measurements are compared to Monte Carlo (MC) simulations in the vertical (solid lines) and lateral (dashed lines) direction. B: Dose rate fall of with depth measured on the axis and in 2, 4 and $6 \mathrm{~cm}$ distance. Measurements are again compared to Monte Carlo (MC) simulations.

Measurement uncertainties remain below $5 \%$ and differences to $\mathrm{MC}$ simulations are, except for one value, below $10 \%$. 
A

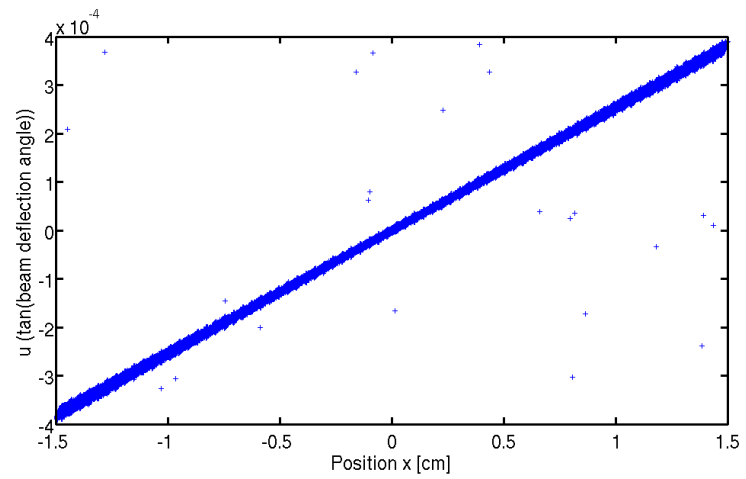

$\mathrm{C}$

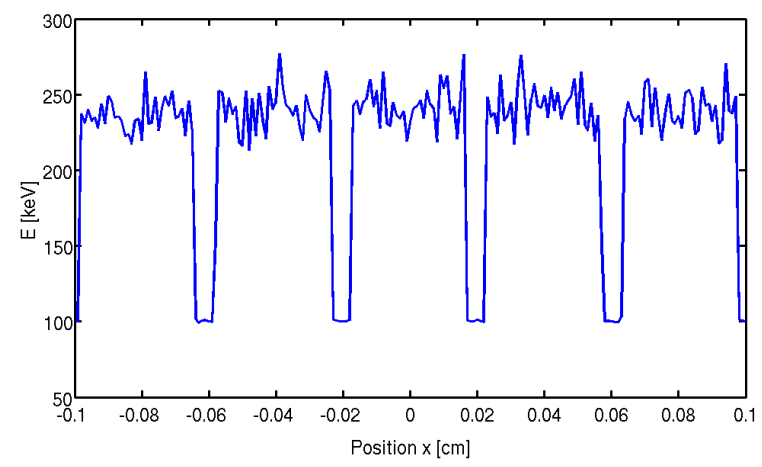

B

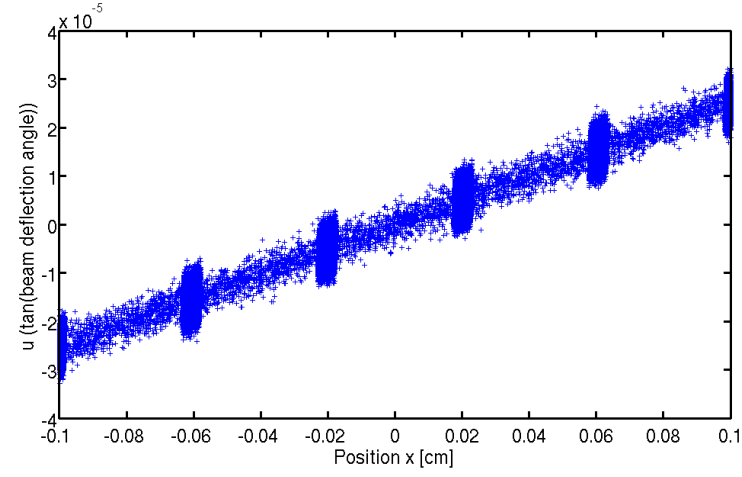

$\mathrm{D}$

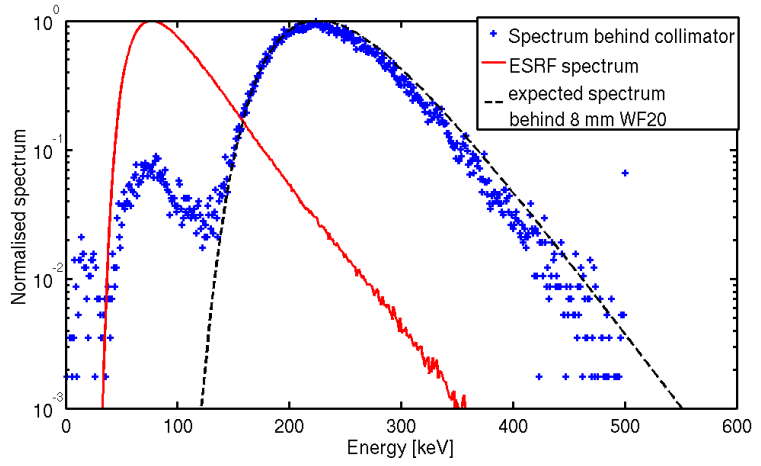

Figure 6 - Analysis of the phase space after tracking the photons from the phase space plane through the MSC. Figure A shows the tangent of the deflexion angle depending on the position in the beam. Figure B shows the same graph in detail at the field centre. Graph

$\mathrm{C}$ plots the mean photon energy depending on the position $\mathrm{x}$. There is a considerable energy difference observable between photons in the beam and between the beams. This is shown in more detail in figure D. As a red line the spectrum in the microbeams is plotted and as blue "'+"' the photon spectrum behind the absorber material. The black dashed line is the expected spectral shift of the red spectrum after the photons penetrated $8 \mathrm{~mm}$ WF20.

A

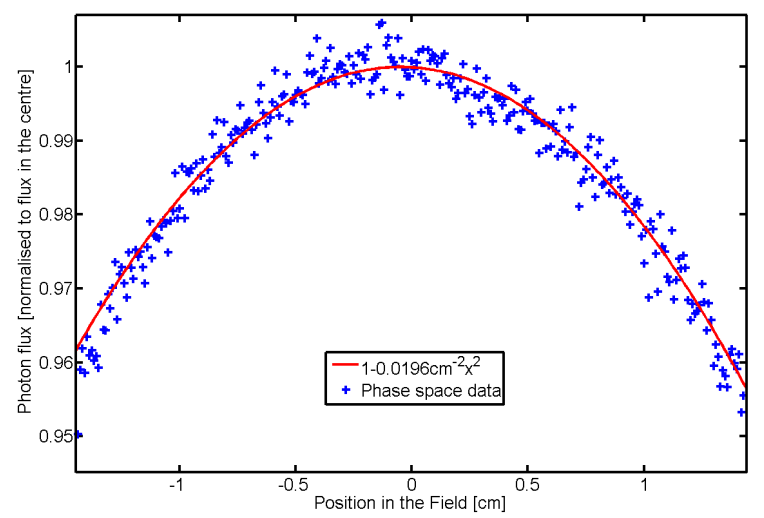

B

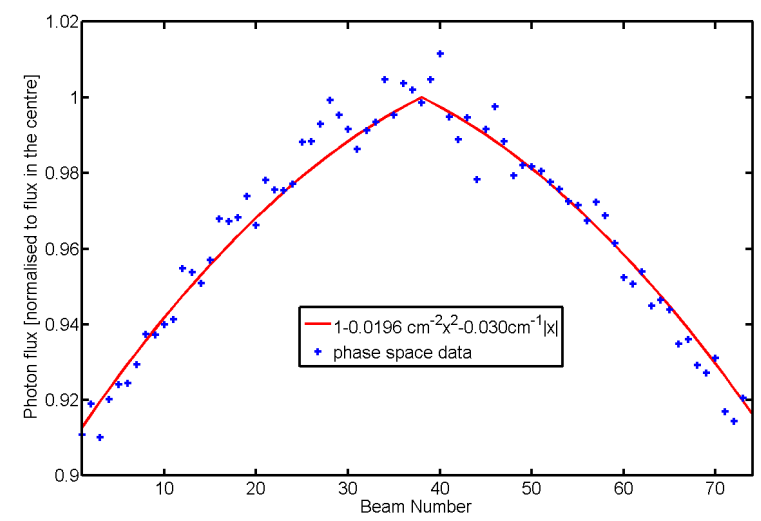

Figure 7 - A shows the photon flux of the primary field in front of the MSC (blue "' + "') and a quadratic quadratic fit (red line) of this data. B shows the relative number of photons per beam (blue "'+") and the developed model (red line). 
A

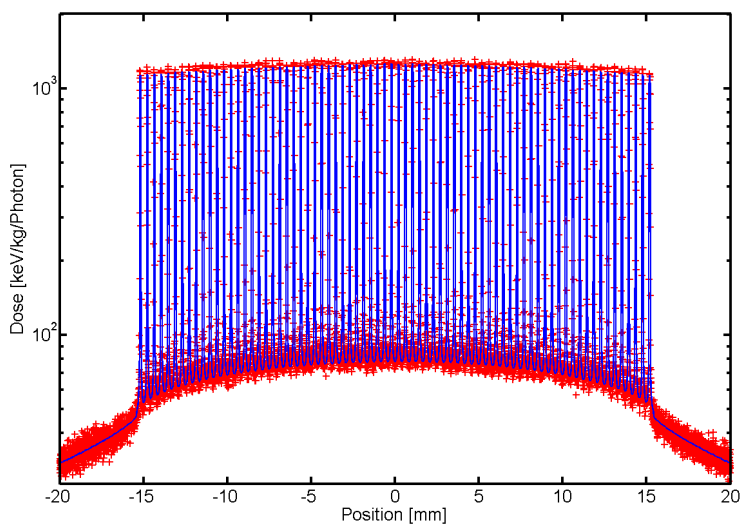

$\mathrm{C}$

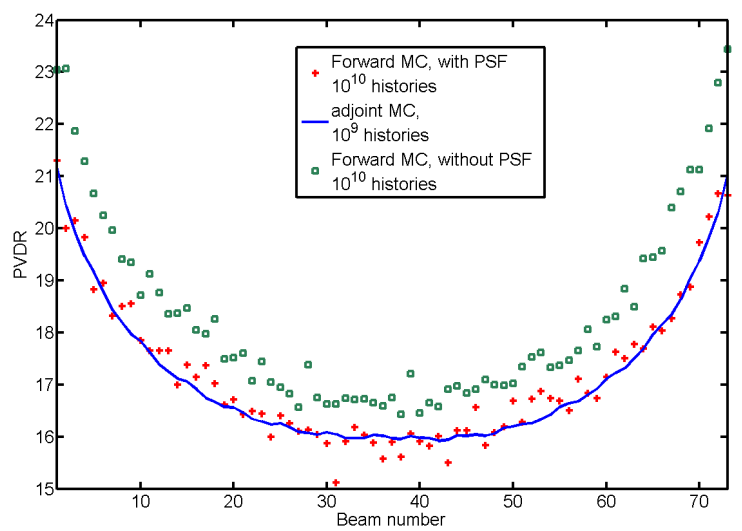

$\mathrm{B}$

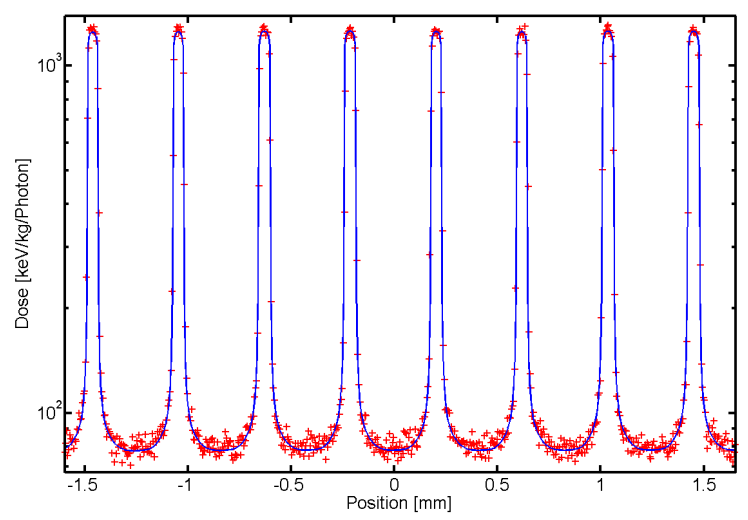

$\mathrm{D}$

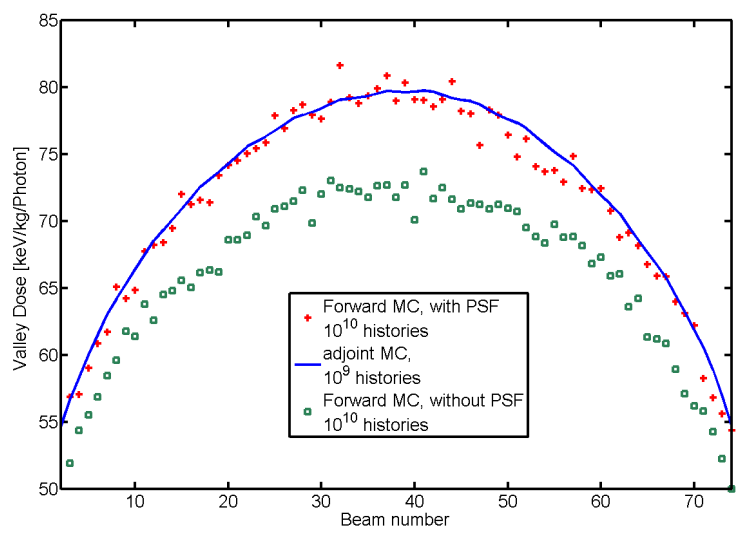

Figure 8 - Comparison of calculations with phase space information (red "“+"), adjoint Monte Carlo calculations with phase space model (blue line) and calculations wihtout phase space information (green "'o"'). A illustrates the whole field and B a few beams in the field centre. In C and D the PVDR and valley dose is plotted over the beam number.

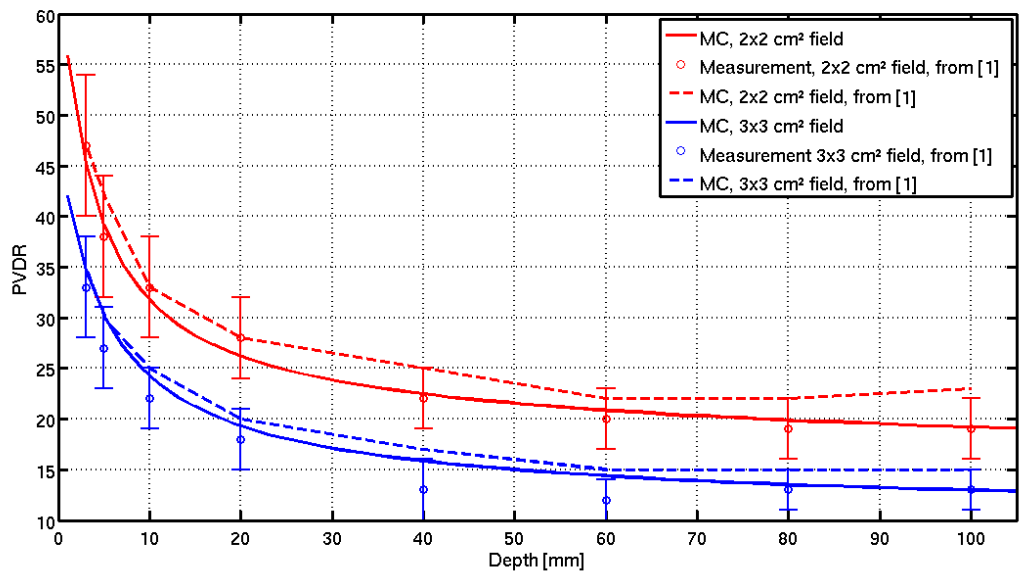

Figure 9 - The graph compares PVDRs in depth calculated with the adjoint Monte Carlo simulation using the model developed in this work (solid line) for a 20x20 mm $\mathrm{mm}^{2}$ and a $30 \times 30 \mathrm{~mm}^{2}$ field in water and compares the result with film measurements (circles) and Monte Carlo simulations (dashed line) by Martínez-Rovira et al. [1]. 